\title{
Trennung radioaktiver Yttererden in einer Adsorptionssäule (Radiometrische Adsorptionsanalyse)
}

\author{
Von Roland Lindner und Otto Peter \\ Aus dem Kaiser-Wilhelm-Institut für Chemie, Tailfingen, und der Forschungsanstalt \\ der Deutschen Reichspost, Miersdorf bei Berlin \\ (Z. Naturforschg. 1, 67-70 [1946]; eingegangen am 10. November 1945)
}

\begin{abstract}
Die Methode der radiometrischen Adsorptionsanalyse (chromatographische Adsorption unter Verwendung radioaktiver Atomarten) wird auf die Yttererden und das chemisch dazugehörige Yttrium angewendet. Hierbei ergeben sich den bisherigen Verfahren überlegene Trennungen dieser Elemente, welche - durch Neutronenbestrahlung radioaktiv indiziert bequem quantitativ bestimmt werden können.
\end{abstract}

$\mathrm{V}_{\mathrm{g}}$ eranlassung zur Einführung der chromatographischen Adsorptionsanalyse ${ }^{1}$ in die kernchemische Methodik gaben spezielle Fragen der Uranspaltung. Nachdem sich bei natürlich radioaktiven Atomarten ${ }^{2}$ und Ceriterden zum Teil überraschend große Trenneffekte gezeigt hatten, lag es nahe, die Versuche auch auf die Yttererden auszudehnen. Bei diesen bekanntlich zum Teil außerordentlich schwer voneinander zu trennenden Stoffen besteht von zwei Seiten ein Bedürfnis nach einem verbesserten Trennverfahren.

1. Die Kernphysik braucht chemische Methoden, um radioaktive Atomarten nach Masse und Kernladung festzulegen, was rein physikalisch (aus der Art der Entstehung, den Eigenschaften der Strahlung usw.) nicht immer möglich ist. Bei den seltenen Erden gab es hierfür bisher keine brauchbaren chemischen Verfahren.

2. Die Chemie fordert im Ausbau ihrer Methodik die Vervollkommnung und Anwendung neuer Trennverfahren und bedarf ihrer - gerade bei den Yttererden - zur Lösung präparativer Probleme.

Die Yttererden, d.h. - in der Systematik nach W. Klemm - die Elemente ${ }_{65}$ Terbium bis ${ }_{71}$ Cassiopeium (s. auch Abb.1) erfordern zur Anreicherung und Reinigung einzelner Glieder bei dem bisher üblichen Fraktionierungsverfahren mitunter Tausende von Arbeitsgängen. Die Anwendung der in der organischen Chemie so gut bewährten Adsorptionsanalyse auf dieses Problem erfolgte zuerst durch Erä mets:ä ${ }^{3}$. Seine bisher zugänglichen

1 Neuste zusammenfassende Beschreibung bei G. Hesse, Adsorptionsmethoden im Chem. Laboratorium (De Gruyter 1944).
Untersuchungen zeigen beachtliche Trenneffekte, doch ist innerhalb des untersuchten komplizierten Erdengemisches infolge geringer Konzentration einzelner Bestandteile die Adsorptionsreihenfolge qualitativ und quantitativ durch die von ihm verwendete Röntgenspektralanalyse nicht immer genau genug festzulegen. Hier liegt nun ein wesentlicher Vorzug radioaktiver Analysenmethoden, die ja, unabhängig von der chemischen Natur, bequeme qualitative und quantitative Bestimmung auf Grund der radioaktiven Eigenschaften gestatten (Aktivitäts-Zeitkurven, verschiedene Härten der Strahlungen).

Hieraus geht hervor, daß die radioaktiven Eigenschaften von Atomarten der untersuchten Elemente eine wesentliche Rolle beim Gelingen der Versuche spielen. Entscheidend ist hierbei die Aktivierbarkeit, da die aktiven Yttererden im Gegensatz zu den ersten Gliedern der Ceriterden nicht bei der Uranspaltung in ,unwägbarer Menge“ anfallen, sondern durch Bestrahlung - meist mit langsamen Neutronen - aus den inaktiven Elementen gewonnen werden müssen. Die Aktivierbarkeit schwankt hier außerordentlich stark (etwa um 4 Zehnerpotenzen), so daß die Versuche, bei denen nur wenige Milligramm Substanz zur Anwendung kamen, nur unter Benutzung einer leistungsfähigen Hochspannungsanlage gelingen konnten, welche durch einen von uns betrieben wurde.

\section{Experimentelles}

Bestrahlt wurden die Erden in wäßriger Nitratlösung (0,2-n.), die dann nach Mischung der zu

2 R. Lindner, Z. physik. Chem. 194, 51 [1944].

3 Olavi Erämetsä u. a., Suomalaisen Tiedeakatemian Toimituksia [Ann. Acad. Sci. fenn.] 57, 3 [1940]. 


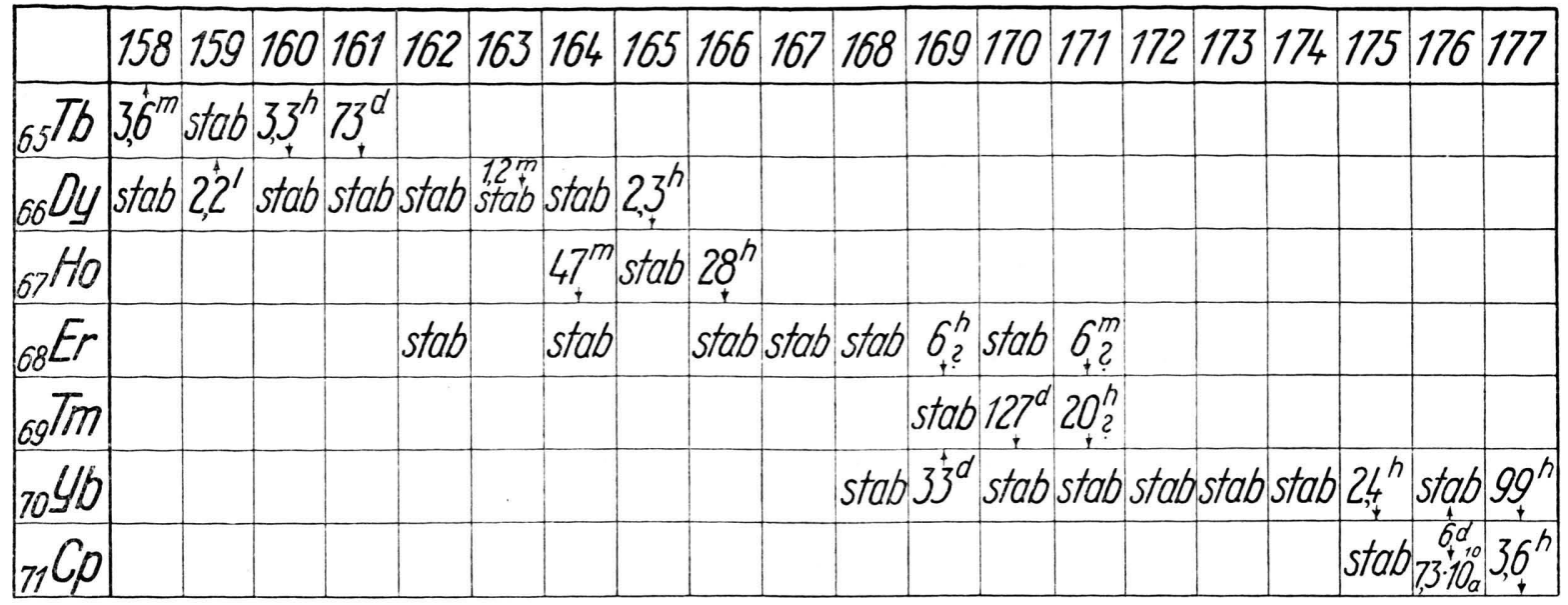

Abb. 1. Isotopentabelle der Yttererden.

untersuchenden Komponenten unmittelbar zur Adsorptionsanalyse gelangte. Die Neutronen wurden aus Lithium mit 1,3 MeV-Deuteronen erzeugt und mit Wasser verlangsamt. Manche Elemente, wie z.B. Dysprosium, konnten auch mit einer um 5 Größenordnungen schwächeren Ra-Be-Neutronenquelle genügend aktiviert werden.

Die Adsorptionstrennung geschah - wie seinerzeit beschrieben ${ }^{2}$ - durchweg an Aluminiumoxydsäulen $\left(\mathrm{Al}_{2} \mathrm{O}_{3}\right.$ nach B r ock m a n $\left.\mathrm{n}\right)$ von $30-40 \mathrm{~mm}$ Länge (bei 5,7 mm Durchmesser) mit 5-10 mg Erden. Allgemein wurden 2 Fraktionen im Filtrat aufgefangen, die Säule ebenfalls in zwei Teile zerlegt und bei diesen vier Fraktionen das Mischungsverhältnis gemessen. Die Erdfraktionen des Filtrates wurden direkt, die der Säule nach Auskochen mit verdünnter Säure und annähernder Neutralisation als Oxalate gefällt (ebenso zur Kontrolle das Ausgangsgemisch).

Die Aktivitätsmessung geschah am 100- $\mu$-AlZählrohr mittels Verstärker in Neher-Harper Schaltung und bis zu 16 -facher Untersetzung. Die Ermittlung der Anteile der zu trennenden Komponenten in den einzelnen Fraktionen erfolgte in bekannter Weise ${ }^{2}$ graphisch aus den Aktivitäts-Zeitkurven. Die mitgeteilten Prozentsätze sind zur gröBeren Anschaulichkeit auf gleiche Ausgangsprozentsätze (50 zu 50) der zu trennenden Komponenten umgerechnet. Zur zahlenmäßigen Kennzeichnung der Trennung wurde zwischen den beiden äußersten Fraktionen der Verteilungsfaktor $\mathrm{Q}=\mathrm{A}_{1} \mathrm{~B}_{4} /\left(\mathrm{B}_{1} \mathrm{~A}_{4}\right)$ gebildet, wobei $\mathrm{A}$ und $\mathrm{B}$ eine und andere Komponente, 1 und 4 erstes Filtrat und oberer Teil der Säule bedeuten.
Ergebnisse

Abb. 1 zeigt eine Isotopentabelle der Yttererden, soweit sie 1944 radioaktiv erforscht waren ${ }^{4}$.

Als allgemeine Voraussage über die Adsorptionsreihenfolge war zunehmende Adsorptionsstärke mit steigender Ordnungszahl, also in der Reihenfolge Terbium-Cassiopeium, anzunehmen. Dies ist verständlich bei rein elektrostatischer Erklärung der Adsorptionsbindung, da ja in dieser Reihenfolge bei gleicher Ladung der Radius der unhydratisierten Ionen stetig abnimmt. Yttrium sollte hiernach in seiner Adsorption wie im Ionenradius zwischen Dysprosium und Holmium erwartet werden. Die Versuche zeigen allerdings, daß diese Regel nicht durchweg gilt, und geben so einen ersten Hinweis auf die Verwendbarkeit der seltenen Erden zur Erforschung des Adsorptionsmechanismus.

1. ${ }_{65}$ Terbium (3,3 Stdn. HZ) wurde nur mit seinem Adsorptionsnachbarn Holmium verglichen und als schwächer adsorbierbar gefunden. Q war 1,6, wobei Terbium im ersten Filtrat von 50 auf $60 \%$ angereichert war.

2. ${ }_{66}$ Dysprosium (2,3 Stdn. HZ) zeigt die erste Ausnahme von oben erwähnter Regel, da es trotz niedrigerer Ordnungszahl stärker als Holmium adsorbiert wird. Drei Versuche zeigten Q-Werte von 1,3; 2,0; 2,7 (maximal 65-proz. Anreicherung des Holmiums im Filtrat), stellten also trotz der Streuung qualitativ diesen Befund einwandfrei fest, der sich auch schon aus der Arbeit Erämetsäs ent-

${ }^{4}$ Neueste Daten nach W. Bothe, Göttinger Akademieberichte 1944, 151-154. 
nehmen ließ (obwohl von diesem nicht besonders darauf hingewiesen war).

3. ${ }_{67}$ Holmium (28 Stdn. HZ) scheint schwächer adsorbiert zu werden als das nächsthöhere Erbium, wenn auch der Trenneffekt für eine sichere Aussage zu klein war.

4. Auch ${ }_{68}$ Erbium (komplex, etwa 10 Stdn. HZ) zeigt noch eine schwächere Adsorption als Dysprosium, das hiernach zwei Plätze höher in der Adsorptionsreihenfolge steht, als sonst zu erwarten wäre $(\mathrm{Q}=2,2)$.

5. ${ }_{69}$ Thulium (127 Tage HZ) endlich wird stärker als Dysprosium adsorbiert (Q-Werte von 1,3 und 1,5, einem 70-proz. Dysprosium im ersten Filtrat entsprechend). Noch größer ist der Effekt gegenüber dem benachbarten Erbium. Hier ergab rechnerische Auswertung aus den Anfangsteilen der Kurven (die abgeschlossen umständehalber nicht mehr zugänglich waren) einen Trenneffekt von 6,0 entsprechend einem 83-proz. Erbium im ersten Filtrat.

6. ${ }_{70}$ Ytterbium (2,4 Stdn. HZ) scheint gegenüber Thulium schwach in der Säule angereichert zu werden; gegenüber dem weiter entfernten Erbium ergab sich eine klare Anreicherung von $Q=4,0$.

7. ${ }_{71}$ Cassiopeium stand damals rein nicht zur Verfügung; ein Versuch, der mit einem mit Cassiopeium verunreinigten Thulium-Präparat durchgeführt wurde, läßt keine Aussage zu.

8. ${ }_{39}$ Yttrium steht in seinen sonstigen chemischen Eigenschaften bekanntlich in der Reihe der Yttererden - etwa zwischen Dysprosium und Holmium. (Hier konnte neben einem durch Neutronenbestrahlung entstehenden 60 -Stdn.-Isotop auch der bei der Uranspaltung entstehende Körper von 57 Tagen $\mathrm{HZ}$ verwendet werden.) Die Angaben Erämetsäs über die Adsorption dieses Elementes sind widersprechend. Einerseits wird für die Adsorption an $\mathrm{Al}_{2} \mathrm{O}_{3}$ aus wäßriger Lösung eine schwächere Adsorption als für alle seltenen Erden (einschließlich Lanthan) angegeben, andererseits soll am sonst gleich wirkenden Silikagel die Adsorption stärker als für fast alle seltenen Erden - etwa so stark wie für Ytterbium - sein. Zur Klärung dieser Frage wurde die Adsorption von Yttrium aus wäßriger Lösung an $\mathrm{Al}_{2} \mathrm{O}_{3}$ mit folgenden Elementen verglichen:

${ }_{57}$ Lanthan: Yttrium wird einwandfrei stärker adsorbiert ( $Q=17$, maximal 90-proz. reines Lanthan im Filtrat). Die Andeutung einer Umstellung dieser Reihenfolge in den ersten beiden Fraktionen, die z. B. bei der Trennung Praseodym-Lanthan ganz klar auftritt, könnte als ,Spitzeneffekt“ der Komplexbildung mit im Adsorptionsmittel vorhandenem Natriumcarbonat gedeutet werden und so auch obigen Befund Erämets äs erklären.

${ }_{59}$ Praseodym: Yttrium wird auch hier noch stärker adsorbiert. ( $Q=3,0$ in zwei Versuchen.)

${ }_{62}$ Samarium aber wird stärker adsorbiert als Yttrium, trotz seiner sonst in der Reihe tieferen Stellung. $(Q=73$. Sowohl Samarium wie Yttrium in den entsprechenden Fraktionen etwa 90-proz. rein.)

Auch ${ }_{63}$ Europium zeigt erheblich stärkere Adsorption $(\mathrm{Q}=42)$ als Yttrium, das auch hier etwa

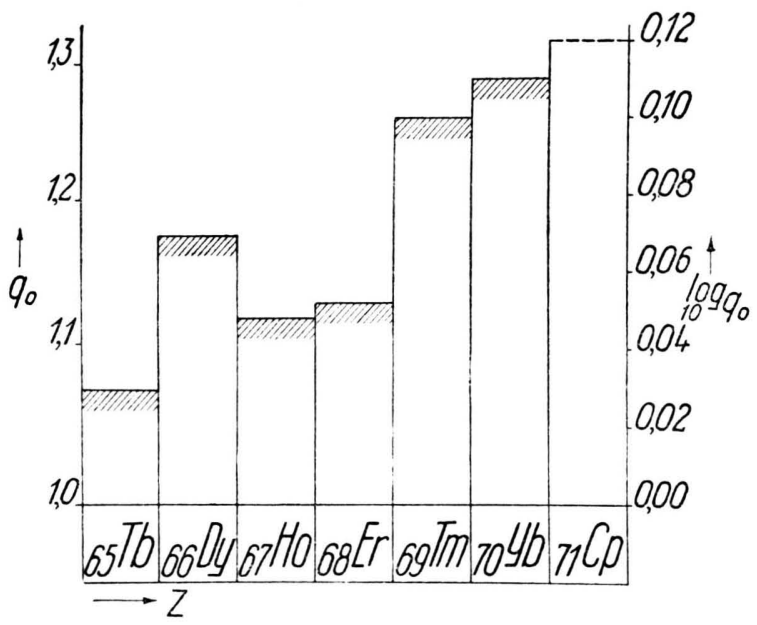

Abb. 2. Adsorptionstrenneffekte der Yttererden gegen ${ }_{30} \mathrm{Y}$.

90-proz. rein im ersten Filtrat auftritt. Ebenfalls stärker als Yttrium werden die sonst dem Yttrium chemisch so ähnlichen Elemente Holmium und Dysprosium adsorbiert (Q-Werte von 5,3 bzw. 7,7; 9,$2 ; 240$ ). (Bei großen Trenneffekten schwanken wegen der willkürlichen Unterteilungen in Fraktionen die Zahlenwerte erheblich.)

\section{Diskussion der Ergebnisse}

1. Hiernach ist Yttrium adsorptionsanalytisch kaum mehr als Yttererde anzusprechen. Die bisher schwierigen Trennungen von Dysprosium und Holmium können, wie das hier zunächst für kleine Mengen gezeigt wurde, verhältnismäßig bequem durchgeführt werden.

2. Für die Reinigung des Dysprosiums kommt vorteilhaft die große Adsorptionsverschiedenheit von den Nachbarn Terbium und Holmium hinzu (s. auch Abb. 2). 
3. Umgekehrt ähneln die letzten Glieder der Ceritreihe wie Samarium und Europium im Adsorptionsverhalten den Yttererden, etwa dem Dysprosium, und können so von den vorangehenden Ceriterden abgetrennt werden.

4. Ähnliche Umstellungen für Yttrium und Samarium finden sich bei von Hevesy ${ }^{5}$ und Britto $\mathrm{n}^{6}$ für die basischen Fällungen (eigene Versuche, die dies $\mathrm{zu}$ bestätigen scheinen und allgemein Aufschluß über den Zusammenhang zwischen Adsorptionseffekt und basischer Fällung geben sollen, sind noch nicht abgeschlossen).

5. Für die kernphysikalische Untersuchung der Yttererden ergeben sich hiernach gute Aussichten einer chemischen Identifizierung, so z. B. im besonders akuten Fall der benachbarten Elemente Erbium und Thulium, die durch das Dazwischentreten von Dysprosium (und wahrscheinlich auch Samarium und Europium) adsorptionsanalytisch gut zu unterscheiden sind.

6. Während so im vorliegenden Adsorptionsfall (bis auf Cassiopeium) die Reihenfolge der Yttererden qualitativ festgelegt gelten darf, zeigen die

5 G. v. Heves y, Die seltenen Erden (S pringer 1927).

${ }^{6}$ H. T. S. B ritt o n, Journ. chem. Soc. [London] 127, $2110-20,2120-41,2142-47,2148-59$ [1925], Chem. Zbl. 1926 I, 735.
Trenneffekte Streuungen infolge willkürlicher Abgrenzung der Fraktionen und ungenügend reproduzierbarer Versuchsbedingungen. Immerhin soll zur vorläufigen Orientierung in Abb. 2 eine ungefähre Darstellung ihrer Größe versucht werden.

Hierbei ist bei den einzelnen Elementen aufgetragen der Logarithmus bzw. der Zahlenwert des Elementar-Trenneffektes $q=Q^{1 / 2}$, der unter der Annahme abgeschätzt ist, daß ein potenziertes chemisches Austauschverfahren ${ }^{7}$ mit 10-20-facher Vervielfachung (experimentell durch eigene Versuche der Trennung von Lanthan und Praseodym sowie von Isotopen nachgewiesen) vorliegt. Als Bezugssubstanz dient das Yttrium. Additive $\mathrm{Zu}$ sammensetzung der Logarithmen und Berücksichtigung der Vervielfachung liefert einen Anhalt für den gesuchten Effekt, wobei die Zulässigkeit dieses Verfahrens zunächst noch dahingestellt bleiben muß.

Die Untersuchung erfreute sich des Interesses von Hrn. Prof. O. Hahn, dem herzlich dafür gedankt sei. Außerdem sind wir Hrn. Dr. Ph. Hoernes von der A u er-A.-G. für die Überlassung hochgereinigter Erdsalze zu Dank verpflichtet. Der eine von uns (R. L.) genoß die Unterstuitzung durch ein Forschungsstipendium des Reichsforschungsrates.

7 H.S. Taylor u. H. C. Urey, J. chem. Physics 8, 429 [1938].

\title{
Über eine Gruppe von Mischphosphoren mit gemischten Aktivatoren
}

\author{
Von Peter Brauer \\ Aus dem Physikalischen Institut der Technischen Hochschule München \\ (Z. Naturforschg. 1, 70-78 [1946]; eingegangen am 1. Oktober 1945)
}

\begin{abstract}
Es wird über Messungen an einer Gruppe von Mischphosphoren mit abnormen Eigenschaften - es handelt sich um Erdalkalisulfidphosphore mit Sm als einem, Ce, Pr, Eu, Mn oder $\mathrm{Pb}$ als zweitem Aktivator - berichtet. Einige leuchten beim Ausheizen und beim Ausleuchten mit völlig verschiedenen Spektren, indem beim Ausheizen nur das Sm+++, beim Ausleuchten nur der andere Aktivator im Spektrum auftritt. Ihre ausheizbare Lichtsumme ist entgegen aller bisherigen Erfahrung kleiner als die ausleuchtbare. Ihre Empfindlichkeit gegen ausleuchtendes bzw. tilgendes Licht ist wesentlich gesteigert. Abweichendes Verhalten eines Gliedes der Gruppe (SrS.Eu-Sm) wird gedeutet und führt zur Aufklärung des Gesamtverhaltens aller Gruppenglieder, damit aber auch - da das Sm sich durchaus in seiner Wirkung als Killer auffassen läßt - eines Falles von Killerwirkung.
\end{abstract}

Tn Fortführung früherer Untersuchungen an 1 Mischphosphoren ${ }^{1}$ wird im folgenden über eine Gruppe von Phosphoren berichtet, die in einigen ihrer Eigenschaften erheblich vom bisher Bekann-

1 P. Brauer, Z. Physik 114, 245 [1939] und Ann. Physik (V) 36, 97 [1939] sowie P. Brauer, Beitrag zur Frage der Energiespeicherung in Phosphoren, in ten abweichen. Anstelle der früher untersuchten Oxydphosphore ${ }^{1}$ handelt es sich dabei jetzt um Erdalkalisulfidphosphore, die gleichzeitig je zwei

„Leuchten und Struktur fester Stoffe", herausgegeben von R. T o m a s ch ek, München und Berlin 1943. - Die vorliegende Arbeit wurde in ihrem experimentellen Teil im Jahr 1942 ausgeführt. 